\title{
Article \\ Plume Divergence and Discharge Oscillations of an Accessible Low-Power Hall Effect Thruster
}

\author{
Matthew Baird, Thomas Kerber, Ron McGee-Sinclair and Kristina Lemmer *
}

check for updates

Citation: Baird, M.; Kerber, T.; McGee-Sinclair, R.; Lemmer, K.

Plume Divergence and Discharge

Oscillations of an Accessible

Low-Power Hall Effect Thruster. Appl. Sci. 2021, 11, 1973. https://doi.org/ 10.3390/app11041973

Academic Editor: Jochen Schein

Received: 23 December 2020 Accepted: 19 February 2021 Published: 23 February 2021

Publisher's Note: MDPI stays neutral with regard to jurisdictional clai$\mathrm{ms}$ in published maps and institutional affiliations.

Copyright: (C) 2021 by the authors. Licensee MDPI, Basel, Switzerland. This article is an open access article distributed under the terms and conditions of the Creative Commons Attribution (CC BY) license (https:// creativecommons.org/licenses/by/ $4.0 /)$.
Mechanical and Aerospace Engineering Department, Western Michigan University, Kalamazoo, MI 49008, USA; matthew.j.baird@wmich.edu (M.B.); thomas.v.kerber@wmich.edu (T.K.); ron.f.mcgee-sinclair@wmich.edu (R.M.-S.)

* Correspondence: kristina.lemmer@wmich.edu

\begin{abstract}
Hall effect thrusters (HETs) are an increasingly utilized proportion of electric propulsion devices due to their high thrust-to-power ratio. To enable an accessible research thruster, our team used inexpensive materials and simplified structures to fabricate the 44-mm-diameter Western Hall Thruster (WHT44). Anode flow, discharge voltage, magnet current, and cathode flow fraction (CFF) were independently swept while keeping all other parameters constant. Simultaneously, a Faraday probe was used to test plume properties at a variety of polar coordinate distances, and an oscilloscope was used to capture discharge oscillation behavior. Plasma plume divergence angle at a fixed probe distance of 4.5 thruster diameters increased with increasing anode flow, varying from $36.7^{\circ}$ to $37.4^{\circ}$. Moreover, divergence angle decreased with increasing discharge voltage, magnet current, and CFF, by $0.3^{\circ}, 0.2^{\circ}$, and $8^{\circ}$, respectively, over the span of the swept parameters. Generally, the thruster exhibited a strong oscillation near $90 \mathrm{kHz}$, which is higher than a similarly sized HET (20-60 kHz). The WHT44 noise frequency spectra became more broadband and the amplitude increased at a CFF of less than $1.5 \%$ and greater than $26 \%$. Only the low flow and low voltage operating conditions showed a quiescent sinusoidal discharge current; otherwise, the discharge current probability distribution was Gaussian. This work demonstrates that the WHT44 thruster, designed for simplicity of fabrication, is a viable tool for research and academic purposes.
\end{abstract}

Keywords: hall effect thruster; discharge oscillation; faraday probe; cathode flow fraction

\section{Introduction}

Hall effect thrusters (HETs) are a subcategory of in-space electric propulsion devices that harness electric energy to accelerate a gas to provide thrust [1]. For in-space applications, the electrical energy is typically acquired by solar panels and stored in batteries. That energy is then converted into charged particle kinetic energy through the use of carefully designed magnetic and electric fields. The primary advantages to utilizing HETs are high propellant exhaust velocities and high thrust-to-power ratios [2]. Exhaust velocities for HETs utilizing xenon propellant are typically between 10 and $20 \mathrm{~km} / \mathrm{s}$, resulting in high specific impulses $\left(I_{s p}\right)$ and thus, increasing the change in velocity $(\Delta V)$ a spacecraft can achieve for a given propellant mass [1]. This enables a spacecraft propulsion system to produce a greater net impulse for a set propellant mass, or, conversely, reduces the necessary propellant for a given mission allowing increased payload mass. Albeit relatively low compared to conventional chemical propulsion, the thrust produced by an HET is sufficient to perform satellite station-keeping, slow orbit transfers, and attitude control maneuvers. Modern mission concepts are even utilizing HETs for deep space exploration missions [3].

The Western Hall Thruster, with a 44-mm outer diameter channel wall (WHT44), was designed as a $200 \mathrm{~W}$ nominal testing platform by an undergraduate capstone group in 2015/16. Details regarding the design and initial characterization experiment can be found in references $[4,5]$, and a brief overview of the design process will be described here. The WHT44 has a channel width of $5 \mathrm{~mm}$ and a channel anode-to-exhaust plane length of 
$14 \mathrm{~mm}$. The goal of the original project was to demonstrate that an HET could be designed, simulated, and manufactured using resources available to a wider number of university students outside of the very high research activity (R1 Carnegie classification), and well funded institutions. The SPT-70 was used as the baseline, and dimensions were initially scaled down before parameterization began in COMSOL Multiphysics software to achieve the desired magnetic lens shape [6]. This desired magnetic lens was achieved by iterating the parametric model in order to emulate the field line shape and centerline strength profile given in reference [1]. The predominate magnetic circuit material and geometry were select to prevent saturation and to minimize cost. The WHT44 discharge anode doubles as both an electrode and as a propellant gas injection manifold. Propellant injected into the discharge channel is done so that the propellant is azimuthally uniform prior to reaching the ionization region of the discharge channel. The magnetic circuit, anode, and discharge channel were machined and assembled in-house at no cost to the project. The WHT44 was completed with a budget of under $\$ 2100$, including an allotment for xenon gas to perform initial characterization testing.

\subsection{Theory of Operation}

\subsubsection{Hall Effect Thruster}

HETs are electrostatic propulsion devices consisting of a hollow cathode, a discharge channel with an anode, and a system to generate a magnetic field radial to the discharge channel. Electrons are inhibited from traveling from the cathode to the anode by the radial magnetic field and, instead, form an azimuthal Hall current in the ExB direction. This Hall current ionizes the neutral propellant injected through the anode into the discharge channel. The more massive ions are mostly unaffected by the radial magnetic field and are instead accelerated by the axial electric field.

While HETs are mechanically simple and classified as electrostatic devices, they rely upon complex plasma physics and magnetic field topologies to operate. HETs exhibit several oscillatory modes over a wide range of frequencies, from $1 \mathrm{kHz}$ to $60 \mathrm{MHz}$ [7]. The effects of these oscillation modes on thruster lifetime, thrust, and efficiency are not fully understood. The two most studied oscillation modes are the breathing mode and the azimuthal spoke mode. The breathing mode is an ionization driven oscillation from the predator-prey relationship between neutral atoms and ions in the plasma plume. According to reference [7], we should expect to see these oscillations at frequencies between $15-35 \mathrm{kHz}$. Breathing mode oscillation frequencies, $f_{i}$, can be modeled in 1-dimension by solving a form of Lotka-Volterra's predator-prey equations, resulting in Equation (1).

$$
2 \pi f_{i}=\frac{\sqrt{u_{i} u_{n}}}{L_{i}}
$$

where $L_{i}$ is the ionization zone length, and $u_{i}$ and $u_{n}$ are the ion and neutral velocity, respectively $[8,9]$. Spoke mode oscillations, when identified with high-speed cameras, are regions of higher plasma light intensity, indicative of an ionization front, most often traveling in the $E \times B$ direction at velocities around $1 \mathrm{~km} / \mathrm{s}[10,11]$.

\subsubsection{Faraday Probe}

Faraday probes are a common diagnostic for determining ion flux in plasma plumes from electric propulsion devices. They are used to quantify thruster beam divergence, identify asymmetric behavior, determine total integrated beam current, and identify various utilization efficiencies (i.e., mass utilization, current utilization, and beam utilization). Faraday probe data can also be collected at a variety of facility background pressures in order to attempt extrapolation of the results to flight-like operation [12]. Fundamentally, Faraday probe data are collected through the use of a planer surface electrode of known area, $A_{p}$, oriented using a cylindrical or spherical coordinate system. A cylindrical coordinate system is one wherein the probe is mounted parallel to the thruster's axial centerline and swept radially. This can be accomplished with either one or two linear stages mounted 
orthogonally with respect to the thrust axis. In a hemispherical coordinate system, the probe is mounted on a rotary stage such that the probe is always facing in the direction of the thruster center point, a point created by the intersection of the thruster discharge exit plane and the thruster centerline. A hemispherical arrangement was used exclusively for this study. The Faraday probe is biased negatively such that it is in the linear ion saturation regime of the plasma. Repeatedly repositioning the probe and collecting data gives the operator a current density contour of the thruster plume [12].

Facility interactions that influence ion current density are present in any ground based experiment. There are two primary interaction mechanisms that exist during ground based testing related to background neutrals in the vacuum facility. First, background neutrals may be ingested into the thruster, ionized, and accelerated, creating excess thrust. Second, charge exchange (CEX) collisions occur when fast ions strike slow neutrals to produce slow ions. CEX collisions scatter beam ions and have the effect of increasing current density in the plume periphery. This results in an increased plume divergence that would not otherwise be present in a space environment. Additionally, corrections due to secondary electron emission (SEE) from the probe collector surface, probe geometry, and thruster distance must be made to avoid excessive error in the analyzed results from Faraday probe data. Reference [12] was utilized substantially for analysis of the Faraday probe data collected in this experiment, and we convey the primary processing methods in the following paragraphs.

Current density, $j$, was calculated by

$$
j=\frac{I_{\mathrm{FP}}}{A_{c}+\kappa_{G}} \kappa_{\mathrm{SEE}}
$$

$\kappa_{G}$ is a correction factor that accounts for probe geometry, and $\kappa_{\text {SEE }}$ is a correction factor that accounts for SEE as a result of ion charge species impacting the tungsten probe surface. $I_{\mathrm{FP}}$ is the current to the Faraday probe, and $A_{\mathcal{c}}$ is the Faraday Probe collector area. An ExB probe (Wein filter) was unavailable for charge species analysis of the WHT44, so the $I_{\mathrm{FP}}$, was assumed to consist only of $\mathrm{Xe}^{+}$. The total beam current, $I_{B}$, and the component of the beam current in the axial direction, $I_{A}$, are calculated using Equations (3) and (4), respectively.

$$
\begin{gathered}
I_{B}=\pi R^{2} \int_{-\pi / 2}^{\pi / 2} j[\theta] \frac{\kappa_{D}}{\kappa_{A}} \sin (\theta) d \theta \\
I_{A}=\pi R^{2} \int_{-\pi / 2}^{\pi / 2} j[\theta] \frac{\kappa_{D}}{\kappa_{A}} \cos \left(\alpha_{A}\right) \sin (\theta) d \theta
\end{gathered}
$$

where $R$ is the radial distance between the Faraday probe and the thruster centerline, and $\alpha_{A}$ is a piecewise function defined as

$$
\alpha_{A}=\left\{\begin{array}{cc}
\theta-\alpha_{N}=\tan ^{-1}\left(\frac{\sin (\theta) \mp \frac{D_{\tau}-W}{2 R}}{\cos (\theta)}\right) & \sin ^{-1}\left(\frac{D_{t}-W}{2 R}\right) \leq \theta \leq 90^{\circ} \\
0 & 0 \leq \theta \leq \sin ^{-1}\left(\frac{D_{\tau}-W}{2 R}\right)
\end{array}\right.
$$

where $\alpha_{N}$ is the probe angle, $D_{\tau}$ is the thruster diameter, and $W$ is the discharge channel width. The beam divergence half-angle, $\lambda$, is defined as

$$
\lambda=\cos ^{-1}\left(\frac{I_{A}}{I_{B}}\right),
$$

and $\kappa_{D}$ and $\kappa_{A}$ are correction factors for measuring ions coming from a two-point source in a hemispherical coordinate system. $\kappa_{D}$ corrects for the difference in radii formed due to the fact that the thruster annulus is not an ideal point source. $\kappa_{A}$ corrects for the change in incidence angle of ions impacting the probe face as angle, $\theta$, and radius are swept. There is a second method for calculating $\lambda$ based on the angle that includes $90 \%$ (or $95 \%$ ) of the total ion current. To maintain consistency between the methods of analysis for the Faraday 
probe data, Equation (6) was used in this work. The difference between the two methods at varying background pressure and thruster diameter downstream (TDD) was examined for the WHT44 in reference [13]. The method outlined using Equation (6) consistently showed smaller half-angles compared with the method that uses $95 \%$ of the total ion current by $\approx 10^{\circ}$.

Faraday probe data were used to determine the beam current and the axial component of the beam current using Equations (3) and (4), from which three thruster loss mechanisms are determined. These are the current utilization efficiency, $\eta_{C}$, mass utilization efficiency, $\eta_{M}$, and beam utilization efficiency, $\eta_{B}$ defined with Equations (7)-(9).

$$
\begin{gathered}
\eta_{C}=\frac{I_{b}}{I_{d}} \\
\eta_{\mathrm{M}}=\frac{I_{\mathrm{B}}}{\dot{m}_{T} Q} \frac{M_{i}}{e} \\
\eta_{\mathrm{B}}=\cos ^{2}(\lambda)
\end{gathered}
$$

where

$$
Q=\left(\sum_{k=1} \frac{\Omega_{k}}{Z_{k}}\right)^{-1}
$$

$\eta_{C}$ represents the ratio of useful ion beam current to the total discharge current, $\eta_{M}$ represents the ratio of the ion mass flow to the total propellant mass flow consumed by the thruster, $\eta_{B}$ quantifies the component of the momentum lost due to the plume divergence, $M_{i}$ is the ion mass, and $\Omega_{k}$ and $Z_{k}$ are the ion current fraction and charge of the $k^{\text {th }}$ species, respectively.

The Faraday probe used for this study had a collector electrode made of tungsten with a diameter of $6.33 \mathrm{~mm}$. The gap distance between the collector and the guard-ring measured $0.835 \mathrm{~mm}$. The guard-ring had an outer diameter of $13 \mathrm{~mm}$ with the overall depth of the collector and guard-ring being $10 \mathrm{~mm}$. The probe was biased to $-15 \mathrm{~V}$ based off of extensive Langmuir probe sweeps that demonstrated ion saturation to occur below $0 \mathrm{~V}$ in the plume, as shown in Figure 1.

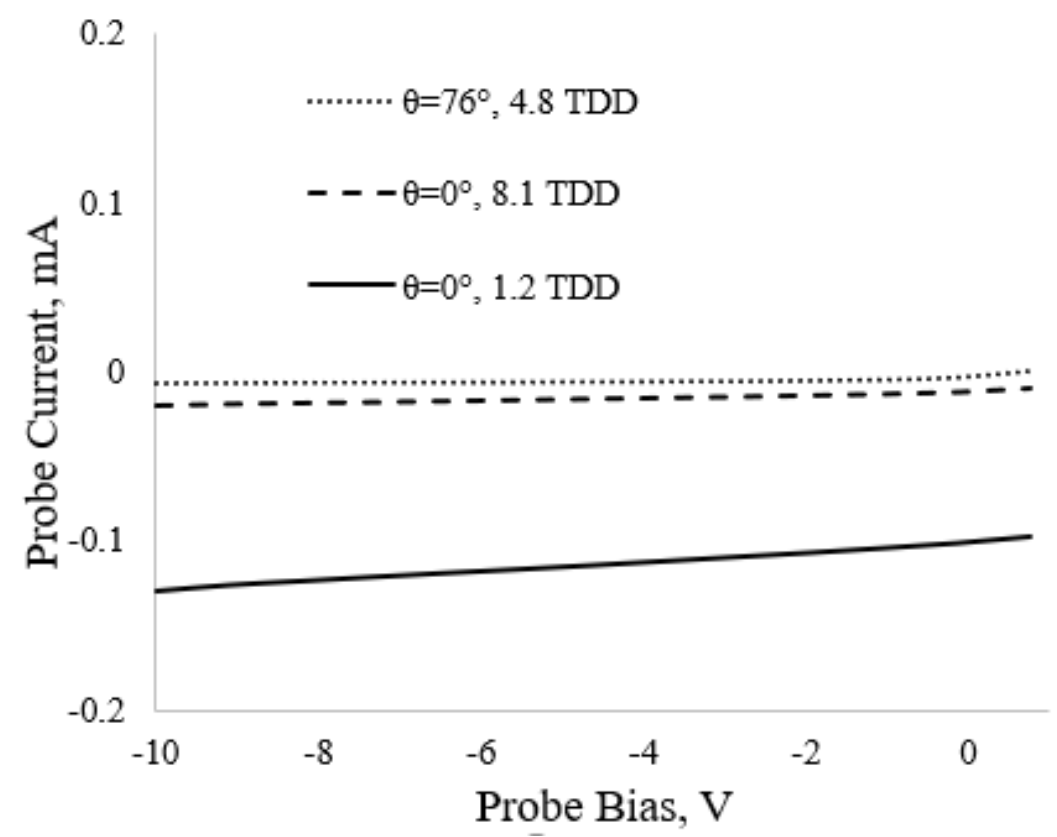

Figure 1. Langmuir probe I-V traces for 44-mm-diameter Western Hall Thruster (WHT44) at $200 \mathrm{~W}$ and several plume locations. 


\section{Materials and Methods}

The study presented in this manuscript was a sweep study wherein a single thruster parameter was adjusted while holding all other control variables constant and observing the effects. Notably, changes in the the thruster discharge current, high-speed behavior, and plume measurements were recorded at every operational data point. This study was performed over two vacuum chamber evacuations. Under the first evacuation, three sweep studies were performed:

- Discharge voltage was swept from $170 \mathrm{~V}$ to $210 \mathrm{~V}$ while holding magnet current to $200 \mathrm{~mA}$, anode mass flow rate, $\dot{m}_{a}$, to $1.27 \mathrm{mg} / \mathrm{s}$, and using a cathode flow fraction (CFF) of $20.7 \%$

- Discharge voltage was swept from $170 \mathrm{~V}$ to $220 \mathrm{~V}$ while holding magnet current to $200 \mathrm{~mA}, \dot{m}_{a}=1.08 \mathrm{mg} / \mathrm{s}$, and using a cathode flow fraction of $20.7 \%$

- Magnet current was swept from $200 \mathrm{~mA}$ to $230 \mathrm{~mA}$ while holding discharge voltage to $200 \mathrm{~V}, \dot{m}_{a}=1.27 \mathrm{mg} / \mathrm{s}$, and using a cathode flow fraction of $20.7 \%$

CFF is the ratio of the cathode mass flow rate to the anode mass flow rate, $\dot{m}_{c} / \dot{m}_{a}$.

Under the second evacuation, the CFF was swept from $1.5 \%$ to $30.8 \%$ while holding discharge voltage to $170 \mathrm{~V}$, magnet current to $200 \mathrm{~mA}$, and xenon anode flow to $1.27 \mathrm{mg} / \mathrm{s}$. The $170 \mathrm{~V}$ discharge voltage on the second evacuation was selected to support a concurrent study. A complete summary of the parameterization study and the resulting power levels are shown in Table 1. Parameter limits were selected to keep the total discharge power below $300 \mathrm{~W}$ or to keep the thruster from extinguishing, whichever came first. The remainder of this section will describe the experimental facility, test equipment, and data acquisition system used in this study.

Table 1. Summary of parameterization setpoints with discharge powers highlighted. The $215 \mathrm{~W}$ operating condition (in bold) is designated the nominal operating point.

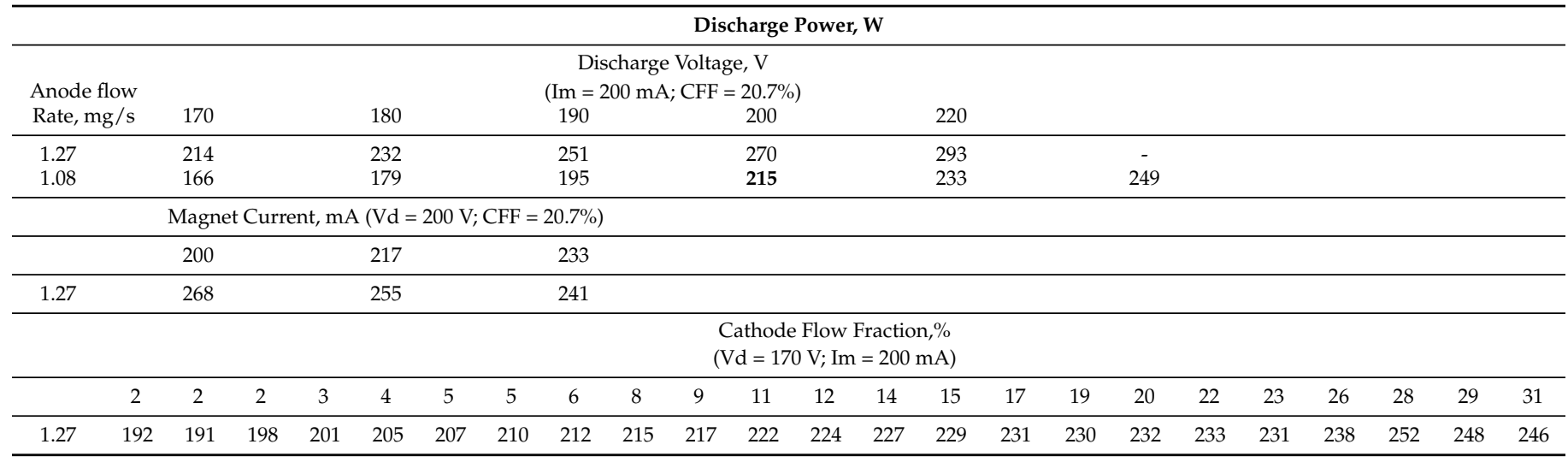

\subsection{Facility}

This experiment was performed in the Aerospace Laboratory for Plasma Experiments' (ALPE's) vacuum chamber facility at Western Michigan University. The facility is a 1-mdiameter, 1.5-m-long stainless steel chamber. A single CTI-250F cryogenic pump with a capacity of roughly $2000 \mathrm{l} / \mathrm{s}$ nitrogen was capable of pumping the facility to a base pressure of $1.5 \times 10^{-7}$ Torr- $\mathrm{N}_{2}$. Testing was performed at an anode flow set point of either 1.27 or $1.08 \mathrm{mg} / \mathrm{s}$ using research grade $99.999 \%$ xenon propellant with corresponding facility pressures of $6.1 \times 10^{-4}$ and $8.4 \times 10^{-4}$ Torr-Xe, respectively. Three laboratory power supplies provided power to the magnets, discharge, and cathode keeper. Xenon flow was controlled to the discharge anode and cathode by two Alicat MCV mass flow controllers calibrated for xenon. Chamber pressure was monitored by a single xenon calibrated ionization gauge. Figure 2 shows a simplified diagram of the experimental setup. 


\subsection{Thruster}

As previously mentioned, two anode flow set points of 1.08 and $1.27 \mathrm{mg} / \mathrm{s}$ were used. A 1.6-mm diameter heaterless hollow cathode with a tungsten-ceramic composite insert from Plasma Controls, LLC. was mounted orthogonal to the WHT44. The cathode was mounted at the twelve-o-clock position oriented downward such that the cathode keeper orifice was $30 \mathrm{~mm}$ above thruster centerline and $11.6 \mathrm{~mm}$ forward of the discharge channel exit plane. This placed the orifice of the cathode inside of the separatrix of the magnetic field. The thruster was operated with a CFF of $20.7 \%$ for the varying discharge voltage and varying magnetic field experiments. This CFF is higher than the nominal $7 \%$ used for many HETs [14]; however, it was found to be necessary to prevent the thruster discharge from unexpectedly extinguishing. The cathode common and thruster body were electrically tied to ground, as it made thruster startup more consistent. The WHT44 was designed such that the amp-turn ratio of the inner and outer magnet coils allowed for them to be operated in series to generate the desired magnetic field shape. The thruster was located above the vacuum chamber centerline to enable full sweep capability of the Faraday probe $\theta$ axis. Photographs of the WHT44 post-test and operating are shown in Figure 3.

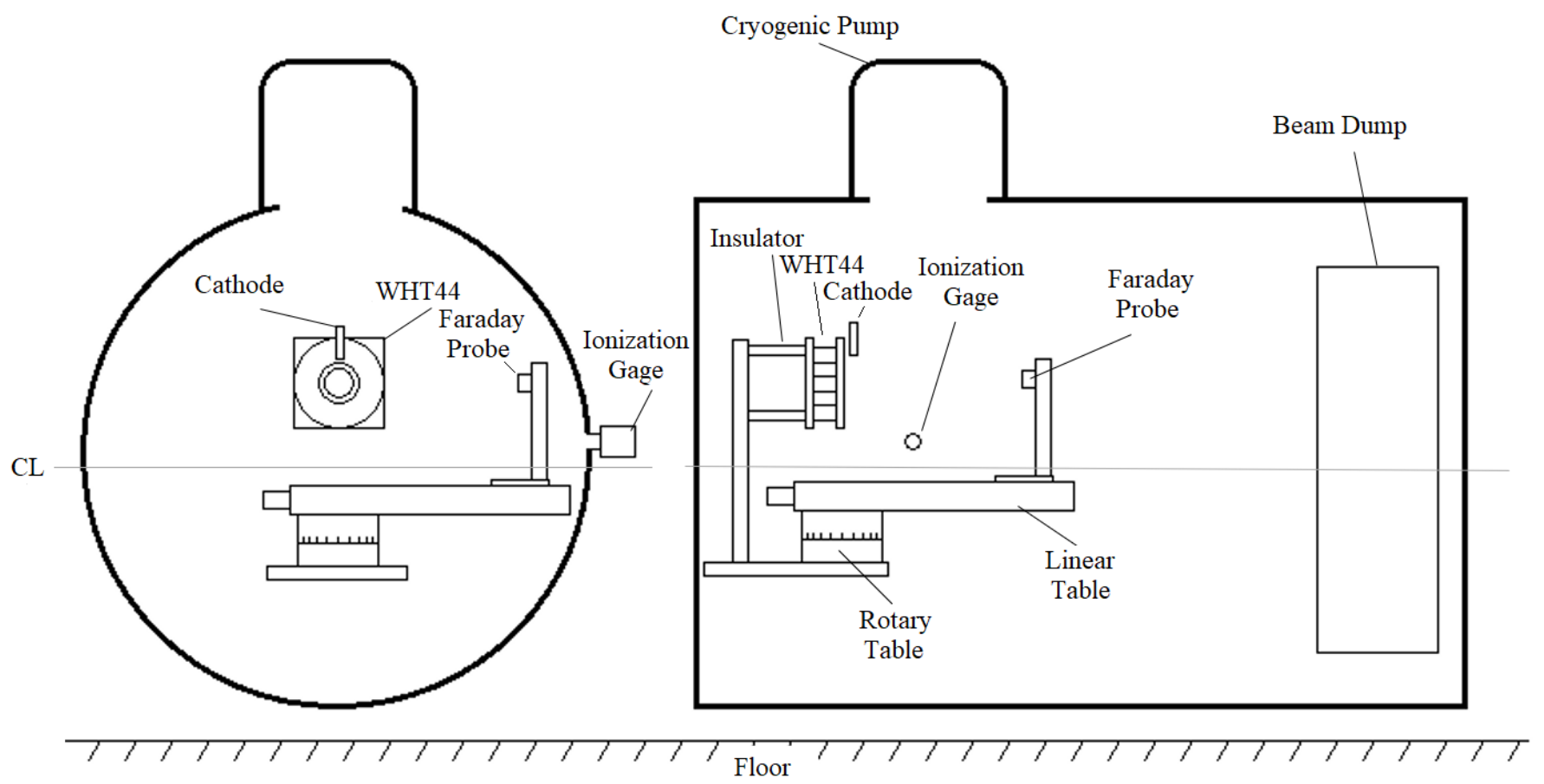

Figure 2. Experimental setup.

Thermocouples were affixed to the WHT44 back pole and on an outer magnet with ceramic adhesive. Maximum temperatures of $252{ }^{\circ} \mathrm{C}$ and $214{ }^{\circ} \mathrm{C}$ were reached at the back pole and outer magnet, respectively. For the duration of testing, cathode keeper current was regulated at $300 \mathrm{~mA}$ for added thruster stability and to reduce thruster extinguishing events. Mean discharge current was obtained using a shunt resistor and custom AD210 isolation amplifier sampled at approximately $1 \mathrm{~Hz}$ with an Agilent 34980A data acquisition (DAQ) mainframe digital multimeter and 34921A relay mulitplexer. The AC discharge current was monitored using a Pearson coil model 4100 read by a $200 \mathrm{MHz}$ commercially available oscilloscope sampling at $500 \mathrm{MSa} / \mathrm{s}$. 


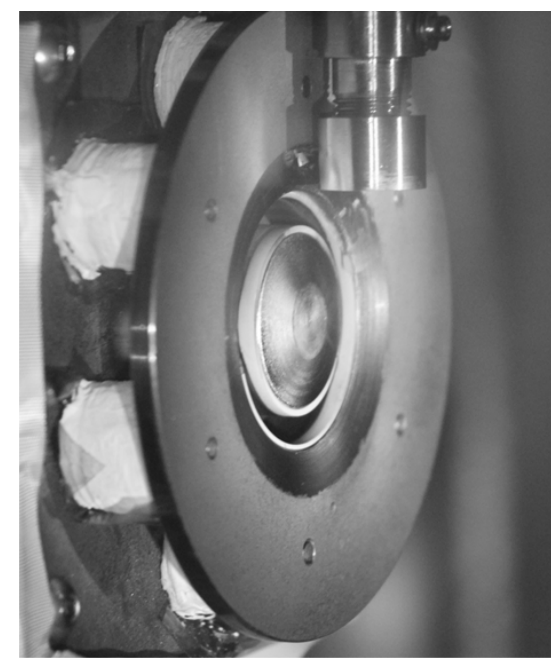

(a)

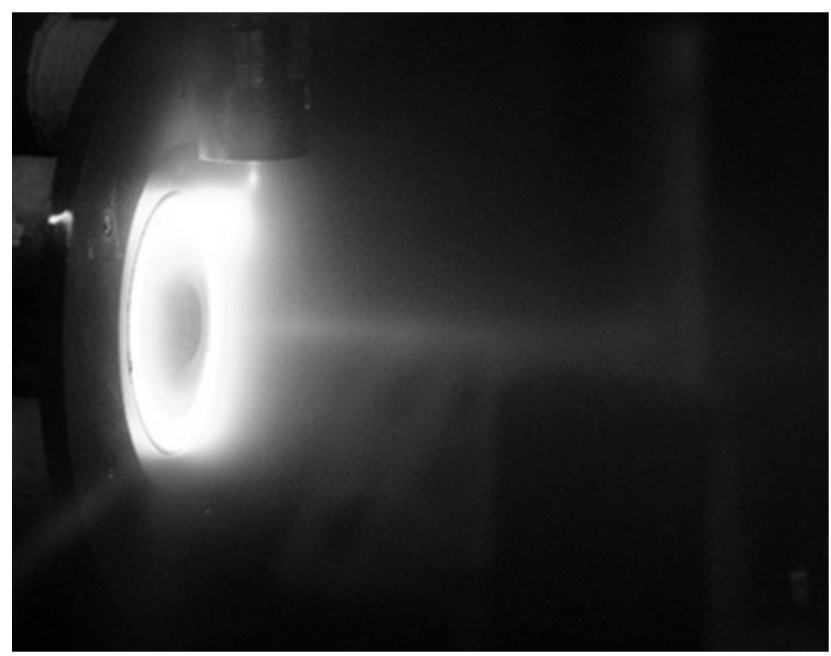

(b)

Figure 3. Photograph of the (a) WHT44 post-test and (b) the WHT44 operating at the nominal operating point.

\section{Results and Discussion}

In this section we share the discharge current behavior and Faraday probe results during the thruster sweep experiments.

\subsection{Thruster Performance}

As previously mentioned, this study was performed during two vacuum facility evacuations separated by removal and reinstallation of the WHT44. Hall thruster performance and testing is typically completed below $1 \times 10^{-4}$ Torr background pressure to minimize the impact on performance [15]. Trends in the results impacted by elevated pressure are highlighted by the authors when discussing the relevant performances. Faraday probe data measured during the first evacuation were recorded at ten radial probe distances ranging from 3.2 to 9 thruster diameters downstream (TDD) and in increments of 0.58 TDD. An example of the FP data collected from the thruster plume at the standard 200-V, 215-W operating point is shown in Figure 4.

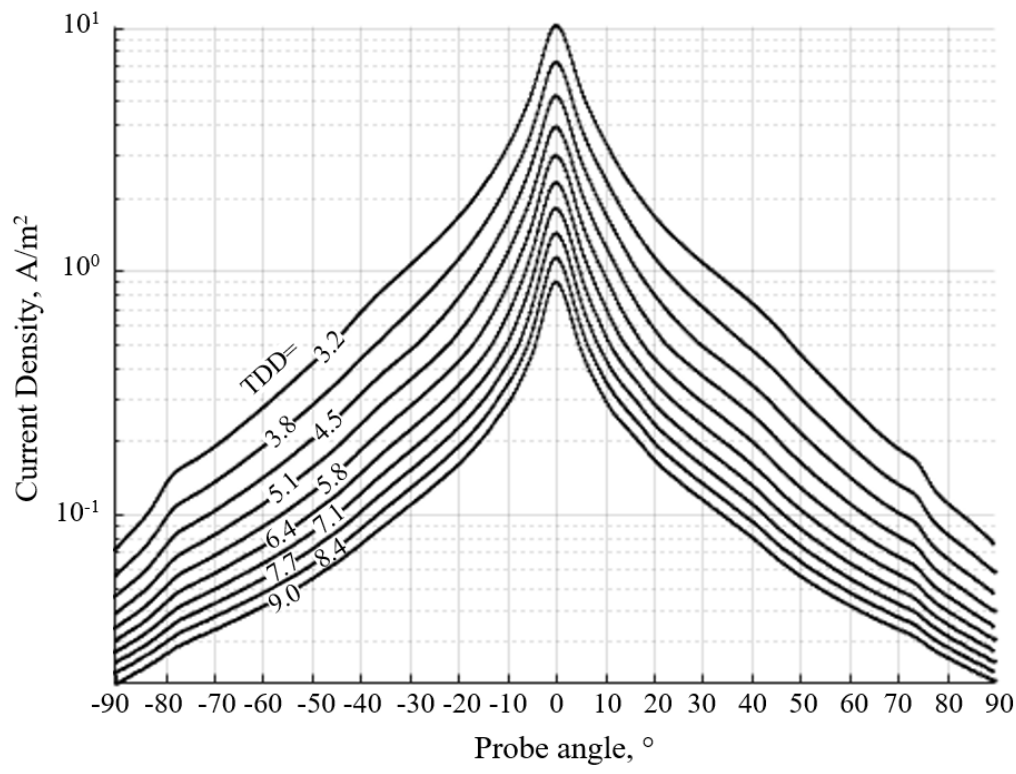

Figure 4. Sample Faraday probe current density measurements at 10 different thruster diameters downstream (TDD) distances plotted together for the nominal 200-V, 215-W operating condition. 
Faraday probe data recorded during the second evacuation were measured at four radial probe distances ranging from $4 \mathrm{TDD}$ to $7.45 \mathrm{TDD}$ in increments of $1.16 \mathrm{TDD}$. While data collection at less than 4 TDD using a hemispherical coordinate system is not recommended, the authors wished to observe trends in the beam properties of the transitionary area between the near and far-field regions of the plume. As a result, data measured at all TDD were analyzed in accordance with the the polar coordinate system methods outlined in reference [12]. Uncertainty in the divergence angle and utilization efficiencies are estimated to have an accuracy of $\pm 5 \%$ based upon estimates calculated by Brown [12].

Total ion current as a function of TDD is plotted in Figure 5. Between 4.5 TDD and 9 TDD, where error analysis is valid, total ion beam current decreases by only 0.0175 A, which is within measurement error margins. This shows total ion current as a function of TDD is conserved, further validating the results from Faraday probe measurements. While the analysis methods used for Faraday probe data lose efficacy in the near plume below 4 TDD the trends can still be useful.

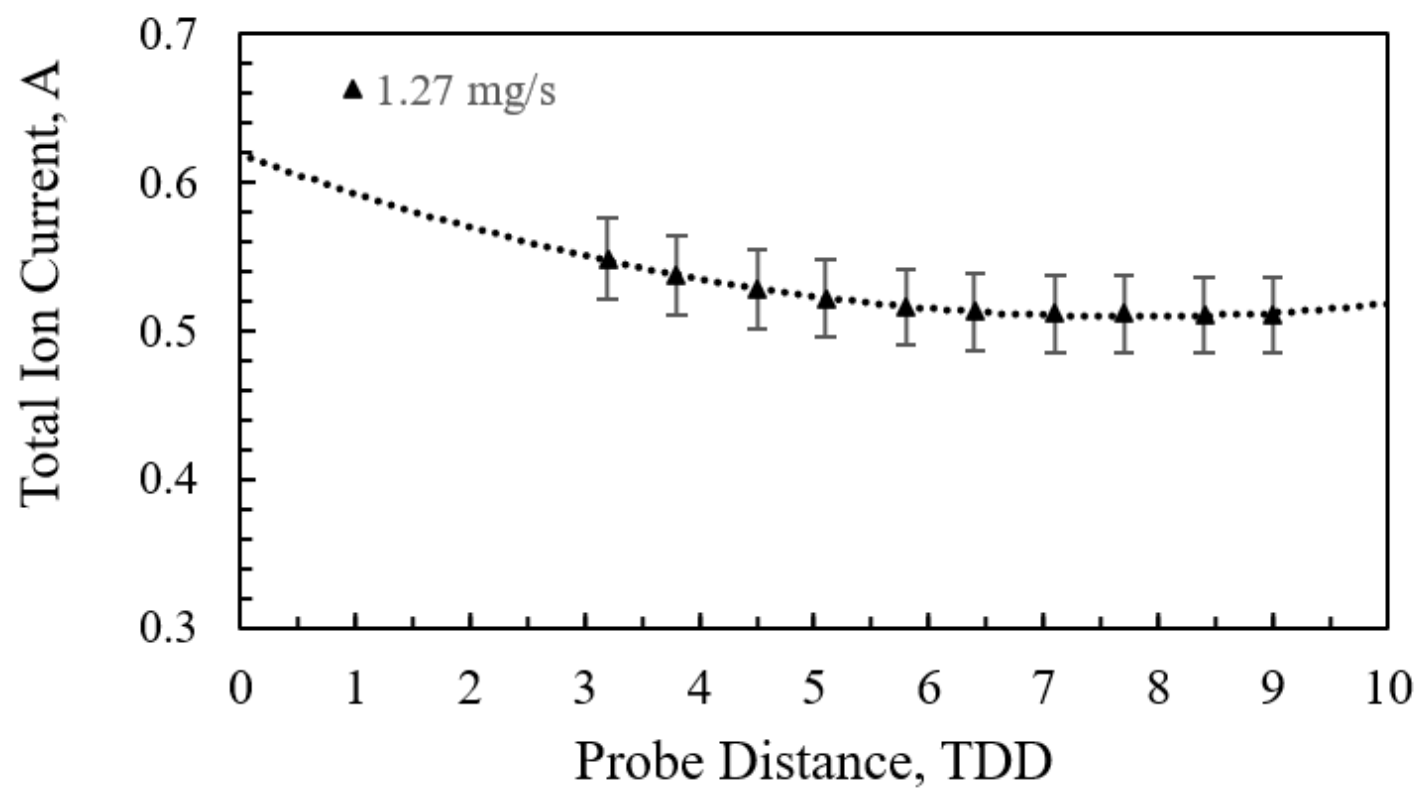

Figure 5. Total ion beam current as a function of TDD at the nominal 200-V, 215-W operating condition.

Thruster divergence results are shown in Figure 6. Firstly, divergence angle as a function of discharge voltage (Figure 6a) and as a function of magnet current (Figure $6 \mathrm{~b}$ at a downstream distance of 4.5 TDD are shown. Next, the divergence angle at the nominal $200-\mathrm{V}, 215-\mathrm{W}$ operating condition as a function of probe distance downstream in TDD lengths is shown in Figure 6c. As seen in other thrusters, the plume divergence half-angle increases as collisions between the plume and background neutrals results in a diffusion of the beam into the chamber [12]. Lastly, the divergence angle as a function of CFF at a probe distance downstream of 4 TDD is shown in Figure 6d. As expected with an HET, the divergence angle decreased with increasing discharge voltage. No significant change in plume divergence angle was detected within the measurement error previously indicated over the full range of discharge voltage, anode flow rate, and magnet current tested. An increase in plume divergence angle of about $9^{\circ}$ was measured over the full range of downstream distances at the nominal 215-W operating condition. These results show divergence angles that are large when compared to other similarly powered HETs $[16,17]$. Both of these trends are likely exacerbated by the high operational pressures. 


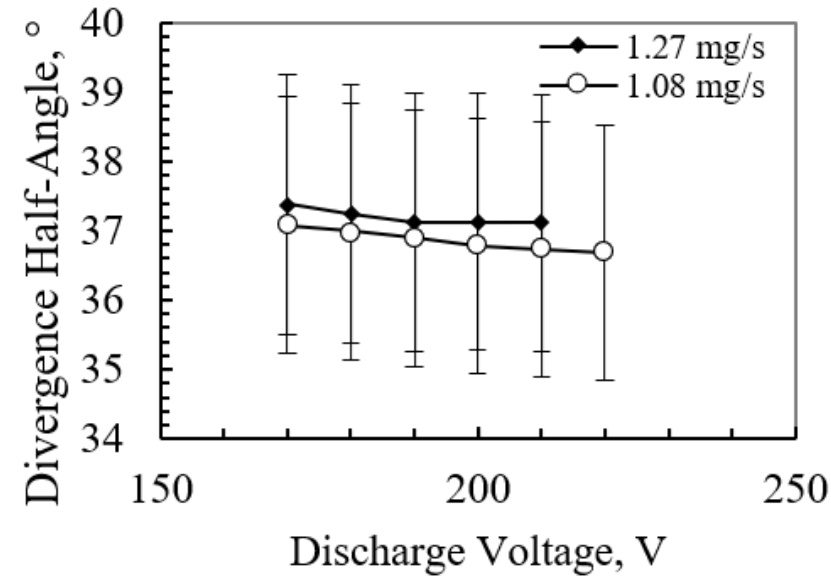

(a)

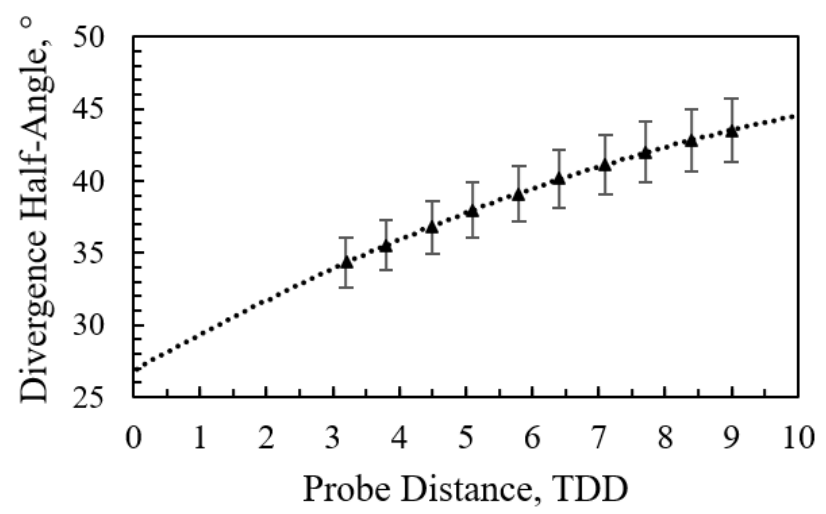

(c)

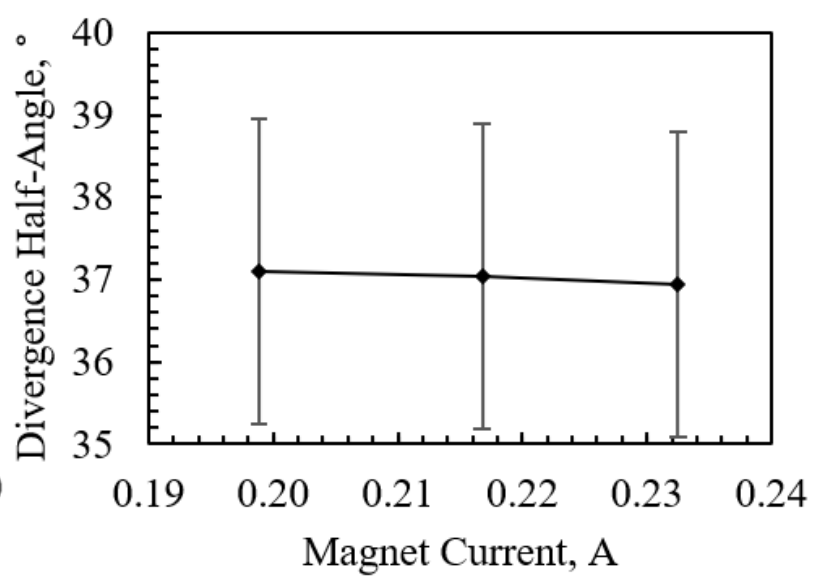

(b)

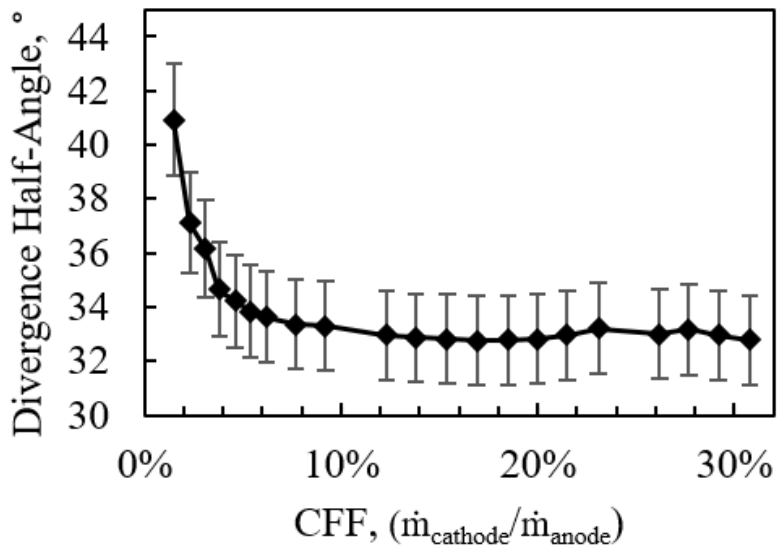

(d)

Figure 6. Divergence angle as a function of (a) discharge voltage, (b) magnet current at 4.5 TDD, (c) probe distance downstream at nominal operating conditions, and (d) of cathode flow fraction (CFF) at 4 TDD.

Utilization efficiencies as a function of discharge voltage and magnet current are shown in Figure 7a,b, respectively. Figure 7c shows utilization efficiencies as a function of CFF at the 200-V, 215-W nominal operating condition. There are several behaviors of note in Figure 7a,b:

- Increased anode propellant flow rate resulted in a higher mass utilization efficiency, likely a result of an increase in the ionization fraction of neutrals within the discharge channel.

- Increased discharge voltage positively affected mass utilization efficiency, and negatively affected current utilization efficiency.

- Increased magnetic field strength positively affected current utilization efficiency,

Taken together, thruster utilization efficiencies shown in Figure 7c show quite poor performance at the lowest CFF operating point of $1.5 \%$ but stabilize to $\eta_{C} \approx 55 \%, \eta_{M} \approx 66 \%$, and $\eta_{B} \approx 68 \%$ by a CFF of $7 \%$. As CFF was increased, the beam utilization efficiency increased and became steady at $7 \%$. Conversely, the mass and current utilization efficiencies spiked in the beginning and then decreased as CFF continued to increase. Current and beam utilization efficiencies, remained relatively constant at all CFF $>7 \%$, which is reflective of a near constant beam ionization fraction and beam divergence. Mass utilization efficiency, naturally decreased due to the relatively constant ion production rate and increasing cathode flow. 


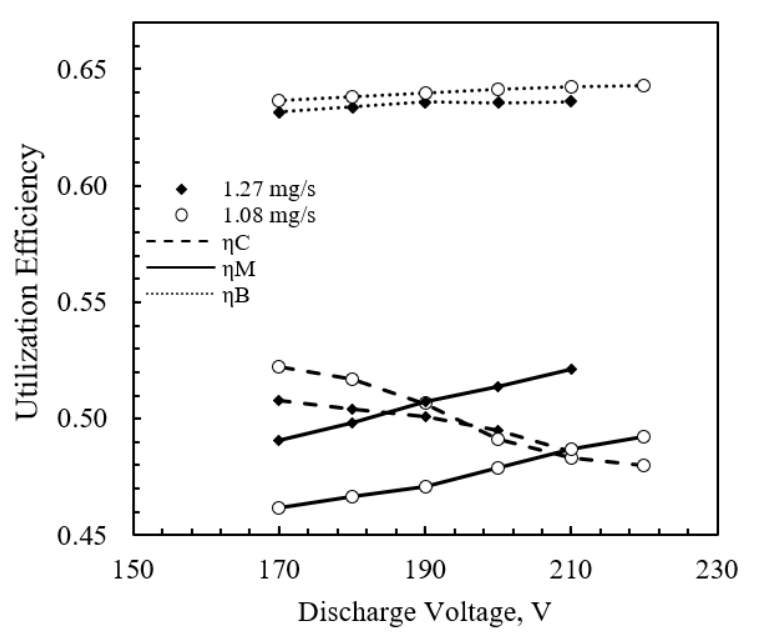

(a)

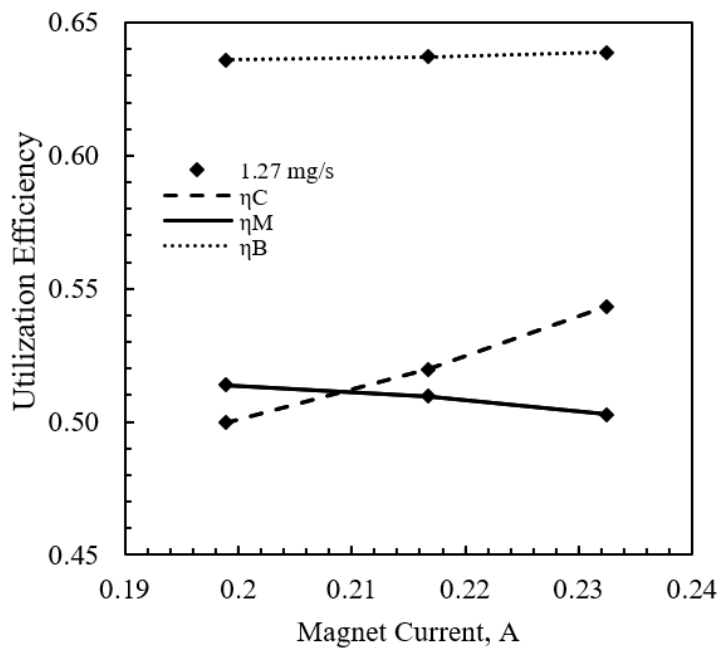

(b)

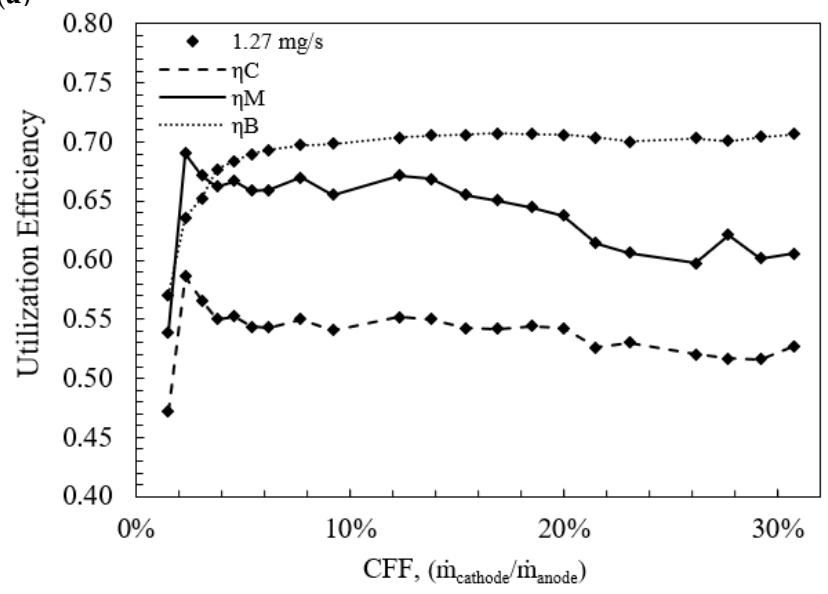

(c)

Figure 7. Current utilization, mass utilization, and beam utilization efficiencies at a probe distance 4.5 TDD as a function of (a) discharge voltage, (b) magnet current, (c) CFF at 4 TDD.

\subsection{Discharge Oscillations}

Discharge current measurements were recorded and processed to determine the mean, peak-to-peak, probability distribution, and spectral density. The mean and peak-to-peak discharge current are plotted as a function of the swept parameters in Figure 8. Figure 8a shows that, in general, discharge current amplitude decreased with increasing discharge voltage. At some operating conditions at the $1.27 \mathrm{mg} / \mathrm{s}$ flow condition, this trend did not hold. The authors cannot fully explain this inconsistency; however, it is likely the manifestation of a complex relationship between wave dampening and changes in the position and length of the ionization zone within the thruster due to an increase in the number of background neutrals. Peak-to-peak discharge current oscillations increased with increasing magnetic field strength, while the mean discharge current decreased as magnetic field strength increased as shown in Figure 8b. Figure 8c shows the mean and average maximum and minimum of the thruster discharge current as a function of CFF at an anode flow rate of $1.27 \mathrm{mg} / \mathrm{s}$. The amplitude of the discharge current oscillations was lowest at a CFF of $6 \%$ and increased sharply at CFFs below $3 \%$ and above $26 \%$. This behavior is consistent with results reported in reference [18] wherein large ion instabilities occur at very low cathode flow rates, and turbulent ion acoustic waves drive increased oscillations at higher cathode flow rates. 


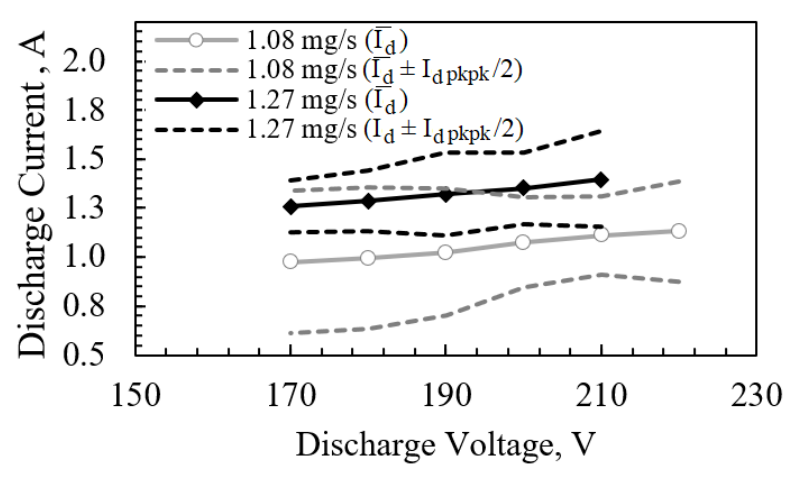

(a)

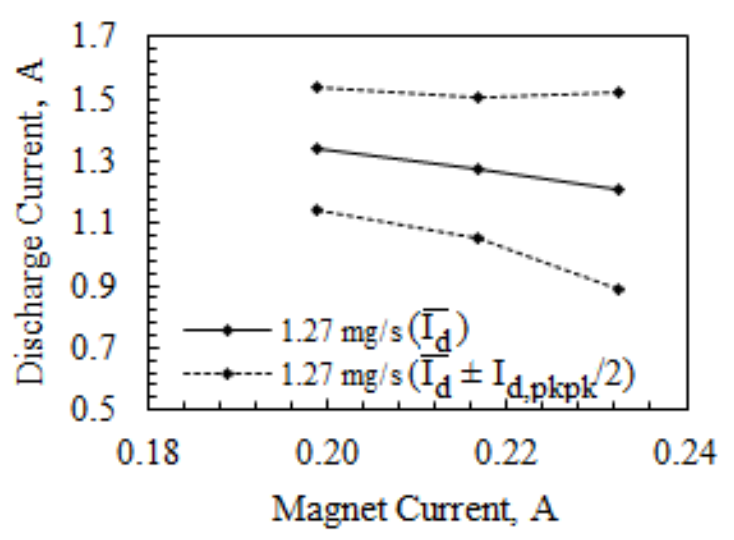

(b)

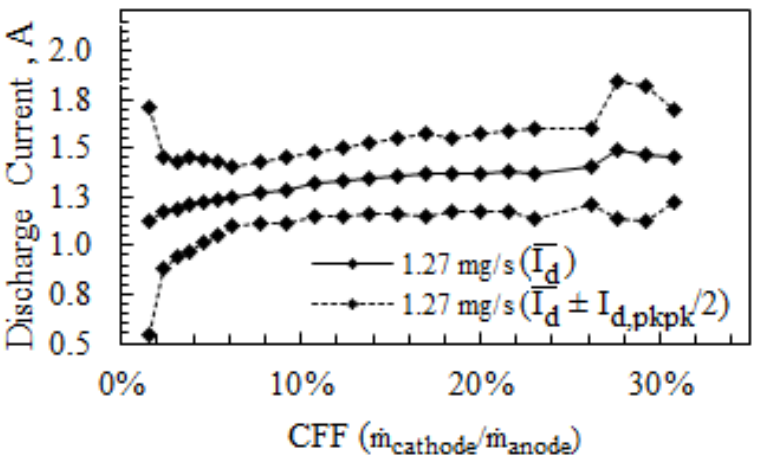

(c)

Figure 8. The mean and peak-to-peak discharge current as a function of (a) discharge voltage, (b) magnet current, and (c) cathode flow fraction.

Utilizing a simple histogram function on the discharge current data, a probability distribution function (PDF) was obtained. The oscillatory behavior of the discharge current at nearly every thruster operating condition exhibited what will herein be referred to as a Gaussian-like probability distribution. An example of this behavior is shown in Figure $9 a, b$ where the thruster is operating at a discharge voltage of $170 \mathrm{~V}$ and an anode flow rate of $1.27 \mathrm{mg} / \mathrm{s}$. Two operating conditions were found to be exceptions to the Gaussian PDF where the discharge current oscillations behaved sinusoidally resulting in a twin-peak PDF, as shown in Figure $9 \mathrm{c}, \mathrm{d}$. The operating conditions that resulted in sinusoidal behavior occurred at discharge voltages of $170 \mathrm{~V}$ and $180 \mathrm{~V}$ while operating at an anode flow rate of $1.08 \mathrm{mg} / \mathrm{s}$. Notably, the sinusoidal discharge behavior corresponds to the highest amplitude in the discharge current oscillations. Thus, there exists a rapid mode shift from a sinusoidal to a Gaussian distribution at an anode flow rate of $1.08 \mathrm{mg} / \mathrm{s}$ between discharge voltages of $180 \mathrm{~V}$ and $190 \mathrm{~V}$.

The power spectral density for each of the swept voltages and magnet currents are shown in Figure 10. The characteristic oscillation frequency varied between 60 and $90 \mathrm{kHz}$ for all operating conditions, which was higher than expected based on other similarly sized thrusters $[19,20]$. As discharge voltage was increased at the high flow condition, Figure 10a, the strongest oscillation frequency remained largely constant at about $60 \mathrm{kHz}$, and the spectral strength remained broadband throughout the range of discharge voltages swept. As discharge voltage was increased at the low flow condition, Figure 10b, the oscillation frequency, initially a strong sinusoidal $60 \mathrm{kHz}$ behavior, decreased to a Gaussian-like oscillation centered at approximately $40 \mathrm{kHz}$. While the sinusoidal behavior appears relatively "clean", there was a greater than normal tendency for the thruster to extinguish under these operating conditions. As magnetic field strength was increased, Figure 10c, the strongest oscillation frequency increased monotonically from about $60 \mathrm{kHz}$ to $85 \mathrm{kHz}$ 
while also increasing in power. The power spectral density as a function of the CFF is shown in Figure 11. At both the highest and lowest CFF, the discharge exhibited a very broadband oscillation varying from $10 \mathrm{kHZ}$ to $150 \mathrm{kHz}$. These extremes in the CFF also corresponded to increases in the peak-to-peak discharge current oscillations. Between the extreme limits of the CFF the discharge oscillation frequency remained near $90 \mathrm{kHz}$ and showed a near constant spectral profile as the CFF increased. Moreover, $6 \%$ CFF is the most spectrally quiescent over the span of CFF tested.

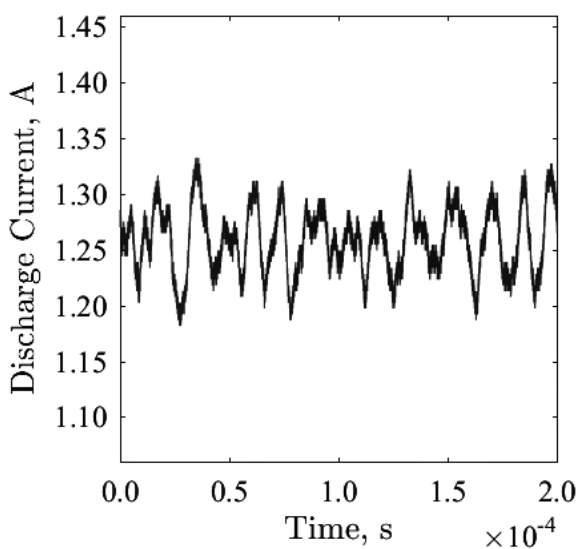

a)

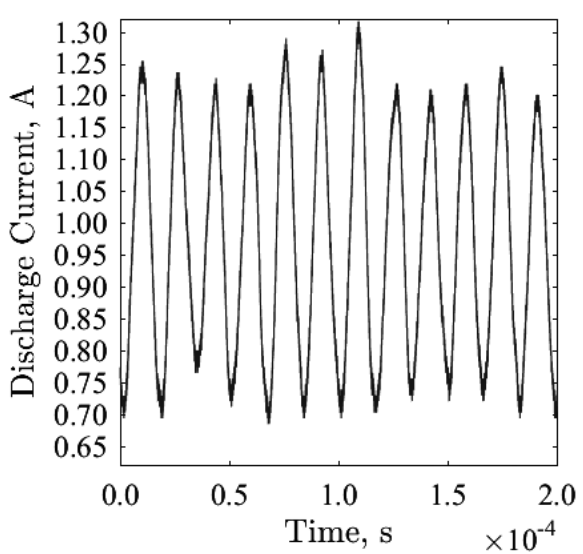

c)

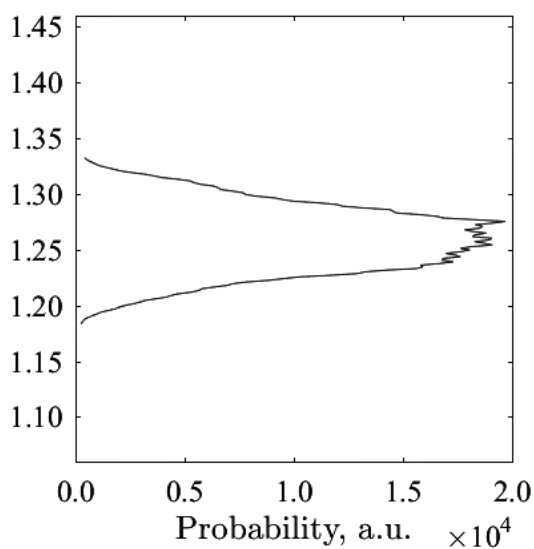

b)

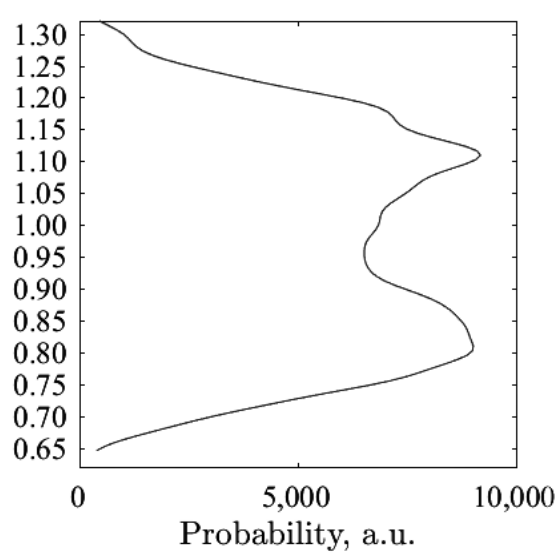

d)

Figure 9. Discharge current as a function of time at $170 \mathrm{~V}$ for mass flow rates of (a) $1.27 \mathrm{mg} / \mathrm{s}$, and (c) $1.08 \mathrm{mg} / \mathrm{s}$. Histograms of discharge current at (b) $1.27 \mathrm{mg} / \mathrm{s}$ setting, and (d) $1.08 \mathrm{mg} / \mathrm{s}$ setting.

Throughout the entire study, the discharge current oscillation frequency largely remained between $45 \mathrm{kHz}$ and $90 \mathrm{kHz}$. This is a surprising result due to the fact that typical breathing mode oscillations occur between $30 \mathrm{kHz}$ and $50 \mathrm{kHz}$ in a similarly sized nonmagnetically shielded thruster [21]. This may be indicative of flow non-uniformities that result in either localized high neutral velocity regions or low characteristic oscillation length, $L_{i}$. Another possible source of these oscillations could be off-axis cathode oscillation modes coupling to the thruster discharge. Future experiments with high-speed imaging could reveal the source of these oscillations. 


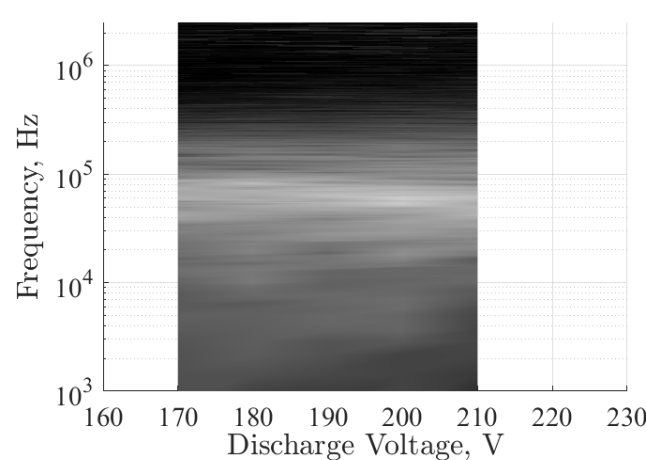

(a)

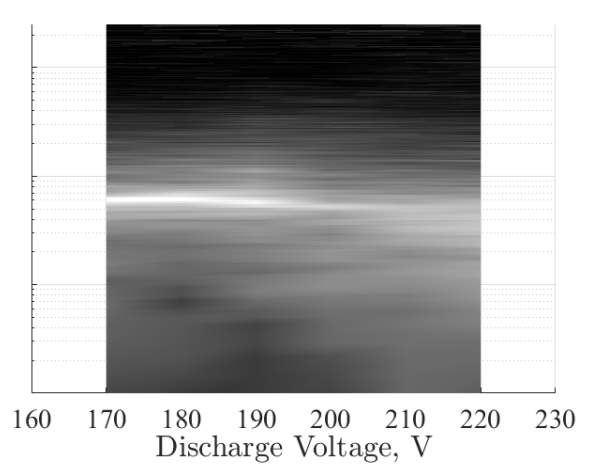

(b)

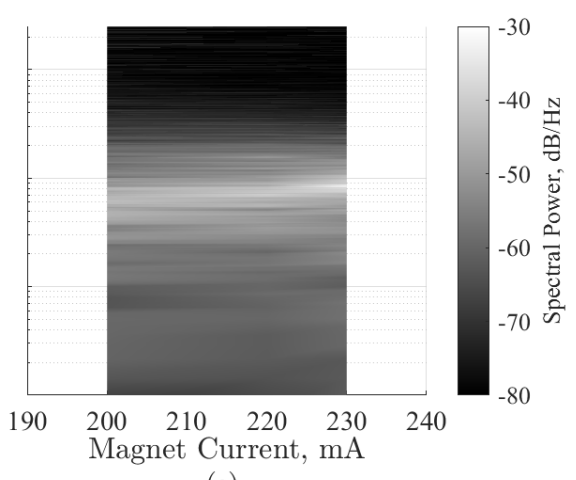

(c)

Figure 10. Thruster discharge power spectral behavior for a CFF of $20.7 \%$ and: (a) $\dot{m}_{a}=1.27 \mathrm{mg} / \mathrm{s}$ and $I_{m a g}=200 \mathrm{~mA}$, (b) $\dot{m}_{a}=1.08 \mathrm{mg} / \mathrm{s}$ and $I_{m a g}=200 \mathrm{~mA}$, and (c) $\dot{m}_{a}=1.27 \mathrm{mg} / \mathrm{s}$ at a $200 \mathrm{~V}$ discharge.

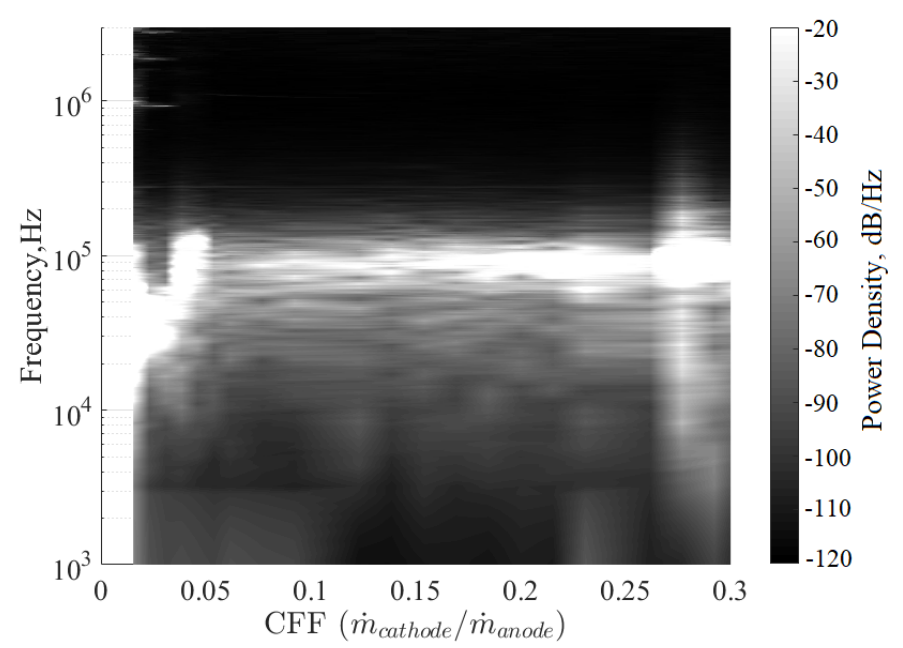

Figure 11. Thruster discharge power spectral behavior for the $170 \mathrm{~V}$ discharge, $\dot{m}_{a}=1.27 \mathrm{mg} / \mathrm{s}$, and $I_{m a g}=200 \mathrm{~mA}$ condition as a function of CFF.

The WHT44 was operated for a duration of $10 \mathrm{~h}$ during this campaign, resulting in a total WHT44 operation time of $42 \mathrm{~h}$. Testing concluded prematurely after a segment of the discharge channel insulator fractured and exposed the front magnetic pole to plasma in the acceleration region. Future plans include making repairs to the discharge channel and continuing with additional plume diagnostics on the WHT44.

\section{Conclusions}

The laboratory model, 200-W WHT44 was examined at an array of operating conditions while sweeping discharge voltage, propellant flow rate, magnetic field strength, and CFF. At each operating point, average telemetry, high-speed discharge behavior, and multiple wide angle FP sweeps were performed. Plume divergence, utilization efficiencies, and discharge current oscillations were quantified showing low thruster efficiencies and a higher than expected dominant oscillation frequency. The WHT44 would benefit from design refinement to increase thermal compliance, increase anode flow uniformity, and conventionalize channel geometry. These changes would likely result in increased ionization zone length, decreased thruster discharge oscillation frequency, and increased stability. Moreover, a study performed on the WHT44 in a facility with higher pumping capacity could greatly expand many of the results reported here.

Author Contributions: Conceptualization, M.B. and K.L.; methodology, M.B. and T.K.; software, M.B. and T.K.; validation, M.B. and K.L.; formal analysis, M.B. and T.K.; investigation, M.B., T.K. and R.M.-S.; resources, K.L.; data curation, M.B.; writing-original draft preparation, M.B., T.K. and R.M.-S.; writing—review and editing, K.L.; visualization, M.B. and T.K.; supervision, K.L.; project 
administration, K.L.; funding acquisition, M.B. and K.L. All authors have read and agreed to the published version of the manuscript.

Funding: The authors would like to thank Western Michigan University's Office of the Vice President for Research for the undergraduate research excellence award used to purchase of all the materials needed for fabrication of the WHT44. This work was supported by a NASA Space Technology Research Fellowship grant number NNX16AM96H.

Data Availability Statement: Data available upon request. Please email Kristina Lemmer at kristina. lemmer@wmich.edu.

Conflicts of Interest: The authors declare no conflict of interest.

\section{References}

1. Goebel, D.M.; Katz, I. Fundamentals of Electric Propulsion: Ion and Hall Thrusters; John Wiley \& Sons, Inc.: Hoboken, NJ, USA, 2008. [CrossRef]

2. Lev, D.; Myers, R.M.; Lemmer, K.M.; Kolbeck, J.; Keidar, M.; Koizumi, H.; Liang, H.; Yu, D.; Schönherr, T.; Gonzalez Del Amo, J.; et al. The Technological and Commercial Expansion of Electric Propulsion in the Past 24 Years. In Proceedings of the 35th International Electric Propulsion Conference, Atlanta, GA, USA, 8-12 October 2017.

3. Brophy, J.R. Legacy of the Asteroid Redirect Robotic Mission (ARRM). In Proceedings of the 35th International Electric Propulsion Conference, Atlanta, GA, USA, 8-12 October 2017.

4. Baird, M.J.; Simmons, N.A.; Lemmer, K.M. Design and Initial Operation of a Small Low-cost Hall Thruster. In Proceedings of the 35th International Electric Propulsion Conference, Atlanta, GA, USA, 8-12 October 2017.

5. Baird, M.J.; Simmons, N.A.; Lemmer, K.M. Performance characterization of a small low-cost Hall thruster. In Proceedings of the 35th International Electric Propulsion Conference, Atlanta, GA, USA, 8-12 October 2017.

6. Comsol. Introduction to COMSOL Multiphysics; 2017.

7. Choueiri, E.Y. Plasma oscillations in Hall thrusters. Phys. Plasmas 2001, 8, 1411-1426. [CrossRef]

8. Fife, J.M.; Martinez-Sanchez, M.; Szabo, J. A Numerical Study of Low-Frequency Discharge Oscillations in Hall Thrusters. In Proceedings of the 33rd AIAA/SAE/ASEE Joint Propulsion Conference, Seattle, WA, USA, 6-9 July 1997. [CrossRef]

9. Sekerak, M.J. Plasma Oscillations and Operational Modes in Hall Effect Thrusters. Ph.D. Thesis, University of Michigan, Ann Arbor, MI, USA, 2014.

10. Boeuf, J.P.; Takahashi, M. New insights into the physics of rotating spokes in partially magnetized e $\times$ B plasmas. Phys. Plasmas 2020, 27. [CrossRef]

11. Mazouffre, S.; Grimaud, L.; Tsikata, S.; Matyash, K.; Schneider, R. Rotating spoke instabilities in a wall-less Hall thruster: Simulations. arXiv 2019, 28, 054002.

12. Brown, D.L.; Walker, M.L.R.; Szabo, J.; Huang, W.; Foster, J.E. Recommended Practice for Use of Faraday Probes in Electric Propulsion Testing. J. Propuls. Power 2017, 33, 582-613. [CrossRef]

13. Kerber, T.V.; Baird, M.J.; Mcgee-sinclair, R.F.; Lemmer, K.M. Background Pressure Effects on Plume Properties of a Low-Cost Hall Effect Thruster. In Proceedings of the International Electric Propulsion Conference 2019, Vienna, Austria, 15-20 September 2019; pp. 1-10.

14. Goebel, D.M.; Jameson, K.K.; Hofer, R.R. Hall Thruster Cathode Flow Impact on Coupling Voltage and Cathode Life. J. Propuls. Power 2012. 28, 355-363. [CrossRef]

15. Kamhawi, H.; Huang, W.; Haag, T.W.; Shastry, R.; Thomas, R.; Yim, J.T.; Herman, D.A.; Williams, G.; Myers, J.; Hofer, R.R.; et al. Performance and Facility Background Pressure Characterization Tests of NASA's 12.5-kW Hall Effect Rocket with Magnetic Shielding Thruster NASA's Space Technology Mission Directorate (STMD) Solar Electric Propulsion. In Proceedings of the 34th International Electric Propulsion Conference, Kobe, Japan, 4-10 July 2015.

16. Brown, D.L.; Larson, C.W.; Beal, B.E.; Gallimore, A.D. Methodology and Historical Perspective of a Hall Thruster Efficiency Analysis. J. Propuls. Power 2009, 25, 1163-1177. [CrossRef]

17. Conversano, R.W.; Goebel, D.M.; Hofer, R.R.; Mikellides, I.G.; Wirz, R.E. Performance Analysis of a Low-Power Magnetically Shielded Hall Thruster: Experiments. J. Propuls. Power 2017, 33, 975-983. [CrossRef]

18. Goebel, D.M.; Jameson, K.K.; Katz, I.; Mikellides, I.G. Potential Fluctuations and Energetic Ion Production in Hollow Cathode Discharges. Phys. Plasmas 2007, 14, 103508. [CrossRef]

19. Conversano, R.W.; Lobbia, R.B.; Tilley, K.C.; Goebel, D.M.; Reilly, S.W.; Mikellides, I.G.; Hofer, R.R. Development and Initial Performance Testing of a Low-Power Magnetically Shielded Hall Thruster with an Internally-Mounted Hollow Cathode. In Proceedings of the 35th International Electric Propulsion Conference, Atlanta, GA, USA, 8-12 October 2017.

20. Grimaud, L.; Mazouffre, S. Conducting wall Hall thrusters in magnetic shielding and standard configurations. J. Appl. Phys. 2017, 122. [CrossRef]

21. Brown, D.L.; Lobbia, R.B. Characterization of Hall Thruster Mode Transitions and Facility Interactions. In Proceedings of the 62nd JANNAF Propulsion Meeting, Nashville, TN, USA, 7-11 December 2015. 